\title{
FACTORES DE RIESGO DE SOBREPESO Y OBESIDAD INFANTIL EN ESCOLARES DE TRES INSTITUCIONES EDUCATIVAS EN EL MUNICIPIO DE CÚCUTA, NORTE DE SANTANDER.
}

\section{RISK FACTORS OF OVERWEIGHT AND CHILDHOOD OBESITY IN SCHOOLS OF THREE EDUCATIONAL INSTITUTIONS OF CÚCUTA, NORTE OF SANTANDER.}

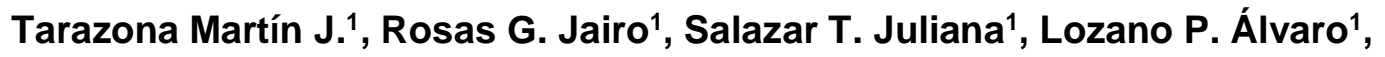

*Wilches R. German²

1. Grupo de Investigación en Salud Publica y Epidemiología, Departamento de Medicina, Universidad de Pamplona. 2. Médico especialista en Salud Ocupacional, Magister en Salud Pública.

Recibido: Julio 24 de 2016; Aceptado 25 de octubre de 2016

\section{RESUMEN}

La obesidad infantil es uno de los problemas de salud pública más graves del siglo XXI, la cual se ha incrementado en los últimos años en Colombia hasta en un 18,9 \%. Con miras a conocer la problemática de nuestra región para poder diseñar estrategias de prevención y control de esta malnutrición por exceso, este trabajo tiene como objetivo Identificar el comportamiento de los factores de riesgos relacionados con el sobrepeso y la obesidad infantil presente en escolares de tercero a quinto grado de primaria del Institución Educativa Gimnasio Domingo Savio, Instituto Técnico Guaimaral, 
Autor a quien dirigirse la
correspondencia: ${ }^{*}$ Wilches Reina
German. Correo electrónico: German. Correo gwilchesr@gmail.com.
Instituto Educativo Pedro Fortoul del municipio de San José de Cúcuta en el periodo 2016-1. Se estudiaron 70 niños entre 8 y 10 años de edad de $3^{\circ}$ a $5^{\circ}$ de primaria de las tres instituciones de Cúcuta. Se tomaron medidas antropométricas y a partir del peso y la talla se calculó el índice de masa corporal para la clasificación nutricional según lo estipula la resolución 2121 del 2010 y se relacionó con las horas de ocio, actividad física y horas de sueño que realizaban los estudiantes, que fueron evaluadas mediante la aplicación de una encuesta. Los resultados muestran que el $23 \%$ de esta población tiene sobrepeso y un 17 \% presenta obesidad. El patrón de actividad física en los niños se caracteriza por actividades sedentarias como ver televisión o juegos de video. El patrón de sueño se observa que la mayoría de los escolares no están durmiendo las horas recomendadas.

Palabras clave: actividad física, horas de sueño, obesidad infantil, tiempo de ocio.

\section{ABSTRACT}

Childhood obesity is one of the most serious problems public health in the 21 st century, since it has been seen that the numbers of overweight and childhood obesity have increased in recent years in Colombia. In order to meet the problems of our region to design strategies for prevention and control of this malnutrition by excess, this paper aims to identify the behavior of the risk factors associated with overweight and childhood obesity present in school Gimnasio Dominic Savio, Technical Institute Guaimaral and Educational Institute Pedro Fortoul at the municipality of San José de Cúcuta in the period 2016-1. We studied 70 children between 8 and 10 years old from 3rd to 5th primary of the three institutions of Cucuta. Anthropometric measurements, height, weight and the BMI were taken for nutritional classification as stipulated in 
resolution 2121 and related to leisure time, physical activity and sleep time were done by the students, which were evaluated by applying a survey. The results show that $23 \%$ of this population is overweight and $17 \%$ are obese. The pattern of physical activity in children is characterized by sedentary activities like using the computer and playing with tablets and smartphones. The sleep pattern is observed that most schools are not getting the recommended hours.

Key words: physical activity, sleep time, childhood obesity, , leisure time.

\section{INTRODUCCIÓN}

La obesidad es una enfermedad caracterizada por un cúmulo de grasa neutra en el tejido adiposo superior al $20 \%$ del peso corporal de una persona en dependencia de la edad, la talla y el sexo debido a un balance energético positivo mantenido durante un tiempo prolongado (1). El exceso de energía se almacena en forma de grasa corporal en los adipocitos que crecen y/o aumentan en número. Esta hipertrofia e hiperplasia de los adipocitos es la lesión patológica característica de la obesidad (2). Su valoración en el niño y adolescente es más difícil que en el adulto, debido a los cambios continuos que se producen en la composición corporal durante el crecimiento. Existen tres períodos críticos para el desarrollo de la obesidad: tercer trimestre de la gestación y primer año de vida, entre los 5 y 7 años de edad y, finalmente, el que corresponde a la adolescencia (3).
El sobrepeso y obesidad es medido por el IMC, pero esta medición no es tan específica en niños como lo es en el adulto, en los niños se tiene que emplear el uso de tablas de patrones de crecimiento infantil para lactantes y niños, digitadas por la OMS y empleadas en la resolución 2121 del 2010 del Ministerio de Protección Social. En estas tablas se usa una puntuación $Z$ donde se permite medir la distancia hacia arriba o hacia abajo en relación con la mediana. La mediana y las puntuaciones $Z$ de cada curva de crecimiento proceden de mediciones de niños del Estudio Multicéntrico de Referencias de Crecimiento de la OMS, quienes fueron alimentados y crecieron en un entorno que favoreció su óptimo crecimiento. Según las desviaciones estándar, si el niño se encuentra entre 1 y menor de 2 se clasifica sobrepeso y una desviación estándar mayor de 2 se clasifica como obesidad (4). 
El sobrepeso y la obesidad son trastornos multifactoriales en los cuales están implicados factores genéticos, de sobrealimentación, reducción de la actividad física, socioeconómicos que contribuyen a su etiopatogenia. Los hábitos nutricionales y el estilo de vida contribuyen sin duda al desarrollo de la obesidad. La ingesta de nutrientes con alto contenido en grasa y poco volumen (dulces, productos precocinados, etc.) llegan a crear un hiper-insulinismo crónico con el consiguiente aumento del apetito, creando una sobre-ingesta y almacenamiento en el tejido adiposo. El Sedentarismo, conlleva una disminución del gasto energético favoreciendo a sí mismo la obesidad. La inestabilidad emocional es otro factor que se asocia al desarrollo de la obesidad ya que conlleva una falta de autoestima lo que comporta: aislamiento, sedentarismo e incremento de la ingesta $(3,5)$.

Dentro de los principales factores de riesgo asociados al sobrepeso y la obesidad descritos en la literatura se encuentran, la disminución del ejercicio físico, originado por un conjunto de circunstancias como los avances tecnológicos en el transporte, entorno urbanístico poco favorable al ejercicio físico, el exceso de consumo de televisión, el cambio de los juegos tradicionales basados en ejercicios físico y de carácter grupal, a juegos electrónicos, que incluso en los últimos tiempos permiten la conexión "on line" entre varios amigos, sin necesidad de desplazamiento alguno se asocia a la aparición de obesidad (6). La cantidad de sueño inferior a 10 horas también es un factor de riesgo para obesidad, por cada hora más de sueño, el riesgo de sobrepeso/obesidad se reduce $9 \%$ (7).

El ambiente socioeconómico tiene una gran importancia en el desarrollo de la obesidad infantil; el estudio epidemiológico de obesidad y sobrepeso de la Dra. Nora Elisabet Zeberio cita que "los pobres no comen lo que quieren, ni saben lo que deben comer, comen lo que pueden. Por lo tanto, están desnutridos porque no tienen lo suficiente para alimentarse y son obesos por que no se alimentan adecuadamente, lo que resulta en un desequilibrio energético importante. Los alimentos que están a su alcance son aquellos productos industrializados de producción barata y masiva" (8).

El sobrepeso y obesidad no son un trastorno único, éste se asocia a un grupo heterogéneo de trastornos que repercuten grandemente en la morbilidad y mortalidad de las poblaciones a través de altas incidencias de diabetes tipo II, hipertensión arterial, enfermedades cerebrovasculares, enfermedades cardiovasculares (IAM), algunos tipos de cáncer y apnea obstructiva del sueño, entre otras $(9,10,11,12)$. 
@.LIMENTECH CIENCIA Y TECNOLOGÍA ALIMENTARIA ISSN 1692-7125. Volumen 14 No. 2, p. 78 -88, año 2016 Facultad de Ingenierías y Arquitectura

Universidad de Pamplona

Las consecuencias de la obesidad son, a corto plazo para el niño o el adolescente: Problemas psicológicos, Aumento de los factores de riesgo de enfermedad cardiovascular, Asma, Diabetes (tipo 1 y 2 ), Anormalidades ortopédicas, Enfermedad del hígado. A largo plazo para el adulto que era obeso de niño o adolescente: Persistencia de la obesidad, Aumento de los factores de riesgo cardiovascular, diabetes, cáncer, depresión, artritis, Mortalidad prematura $(13,14,15)$.

La obesidad es hoy en día considerada una epidemia notoria del siglo XXI (16). Este problema afecta a países de altos, medianos y bajos ingresos; pero los principales afectados son los países de mediano y bajo ingreso ya que están expuestos a un fenómeno llamado "doble carga" de mortalidad. Este fenómeno se debe a que estos países siguen debatiéndose con el problema de las enfermedades infecciosas y la subnutrición, y al mismo tiempo están sufriendo un rápido aumento de los factores de riesgo de enfermedades no transmisibles como la obesidad y el sobrepeso, especialmente en el medio urbano. Por esto, no es raro que en un mismo país, comunidad u hogar coexistan lado a lado la subnutrición y la obesidad (17). En América Latina se identificó que entre 22,2 a 25,9 millones de los niños en edad escolar tiene exceso de peso; de estos el 34,5\% pertenecen a México, el $33,5 \%$ a Brasil y el 18,9 \% a Colombia (18). En
2014, 41 millones de niños menores de cinco años tenían sobrepeso o eran obesos (19).

Esta es la razón principal por la cual es importante tocar este tema ya que la obesidad infantil es uno de los problemas de salud pública más graves a nivel mundial y está afectando progresivamente a muchos países de bajos y medianos ingresos, sobre todo en el medio urbano. La prevalencia ha aumentado a un ritmo alarmante. Se calcula que en 2010 hay 42 millones de niños con sobrepeso en todo el mundo, de los que cerca de 35 millones viven en países en desarrollo (16). Este aumento progresivo tiene múltiples consecuencias debido a que la obesidad infantil se asocia a una mayor probabilidad de muerte y discapacidad prematuras en la edad adulta. Los niños con sobrepeso u obesos tienen mayores probabilidades de seguir siendo obesos en la edad adulta y de padecer a edades más tempranas enfermedades no transmisibles como la diabetes 0 las enfermedades cardiovasculares (20).

Dentro de este contexto, este trabajo tiene como objetivo general identificar los posibles factores de riesgos relacionados con el sobrepeso y la obesidad infantil presente en escolares de tercero a quinto grado de primaria del Colegio Domingo Savio, Instituto Técnico Guaimaral, Instituto Educativo Pedro Fortoul del municipio de San José de Cúcuta en el periodo 2016-1. 


\section{METODOLOGÍA}

La presente investigación corresponde a un estudio descriptivo y observacional, para identificar los factores de riesgo relacionados con el desarrollo de sobrepeso y obesidad, en niño de 8 a 10 años de los grados tercero, cuarto y quinto del Instituto Técnico Guaimaral, Institución Educativa José Aquilino Duran Sede Pedro Fortoul y Colegio Domingo Savio de la ciudad de San José de Cúcuta, en el periodo 2016.

De la población muestra, el universo estuvo conformado por 437 niños entre 8 y 10 años de edad, estudiantes de tercero a quinto grado de primaria de dos colegios públicos y uno privado seleccionados por conveniencia en la ciudad de Cúcuta. Los criterios de inclusión fueron valor de IMC con desviación estándar >-1, que estén matriculados en las instituciones educativas y estén cursando tercero, cuarto y quinto grado de primaria, cuyos padres o personas a cargo aceptaron la participación en el estudio y firmaron el consentimiento informado y los de exclusión todos los niños que tuviesen (necesidades especiales) malformación y/o limitación física y obesidad endógena.

De la población marco (437 estudiantes), se obtuvo una muestra de 142 niños de tercero a quinto de primaria, 47 del Instituto Educativo Pedro Fortoul, 55 del Instituto Técnico Guaimaral y 40 del Instituto
Educativo Gimnasio Domingo Sabio, considerando una prevalencia esperada de sobrepeso de $5 \%(p=0.05)$, con un margen de error de $5 \%$, un nivel de confianza del 95\%; debido a que la mayoría de padres no aceptaron el consentimiento informado el tamaño de la muestra fue de 70 estudiantes, lo que afectó el margen de error.

Se aplicó la técnica de muestreo aleatorio simple, teniendo en cuenta el listado de los estudiantes matriculados en el año 2016 de la jornada de la mañana de las tres instituciones educativas, se identificaron utilizando la calculadora CASIO $f_{X}-350 \mathrm{MS}$, para ello colocamos el número de escolares que componen la población a estudiar, en este caso por cada aula y utilizamos la tecla (Ran\#), obteniendo un numero aleatorio, y se relacionaba con la posición en la lista.

Para la recolección de la información se utilizó una ficha y una encuesta, la ficha contenía los siguientes ítems: Institución a la que pertenece, nombres y apellidos, documento de identidad, edad, sexo, talla, peso, IMC y número telefónico. La encuesta contenía: Institución, nombre, edad, grado, tipificación del estrato por medio de un recibió de servicio de electricidad, hora en la que se acuesta y se levanta el escolar, electrodomésticos presentes en la habitación y su tiempo dedicado diariamente y tiempo 
dedicado semanalmente a la actividad física. Se realizó también valoración de medidas antropométricas.

La talla se tomó la medición sin zapatos, con talones juntos, y para el peso se usó una balanza digital. Antes de iniciar la medición se corroboró que el escolar no tuviera alguna deformidad o discapacidad física. Antes de pesar se verifico que la báscula estuviera en cero. El niño se posicionó en el centro de la plataforma y permaneció inmóvil, con el peso distribuido de forma pareja entre ambos pies, brazos colgados sin ejercer presión, cabeza

\section{RESULTADOS}

La muestra final del estudio quedó constituida por 70 niños de ambos géneros de los cuales 32 pertenecen al Instituto Técnico Guaimaral, 30 Institución Educativa José Aquilino Duran Pedro Fortoul y 8 al Colegio Domingo Sabio. (Ver figura 1).

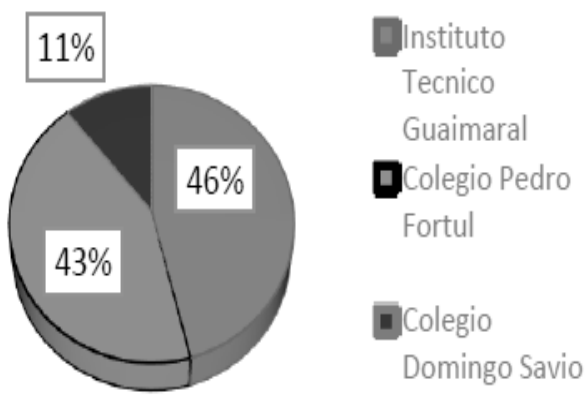

Figura 1. Distribución Institutos Escolares. Fuente: Autores. firme y mirada en un punto fijo, la lectura de la medición se realizó en kilogramos.

El índice de masa corporal se calculó a partir de los datos de peso y talla obtenidos (peso/ talla al cuadrado expresado en $\mathrm{Kg} / \mathrm{m}^{2}$ ). $\mathrm{La}$ clasificación nutricional se obtiene con la resolución 2121 de 2010 en la cual se adoptan los patrones de crecimiento para Colombia publicados por la organización mundial de la salud OMS, en el 2006 y 2007 para los niños, niñas y adolescentes de cero a 18 años de edad.
Dentro de los resultados, aparecen los datos antropométricos de la población total, donde se encontró que el $40 \%$ de los estudiantes tienen malnutrición por exceso (ver tabla 1).

Tabla 1. Diagnóstico nutricional de escolares según IMC.

\begin{tabular}{|l|c|c|}
\hline \multicolumn{1}{|c|}{$\begin{array}{c}\text { Estado } \\
\text { Nutricional }\end{array}$} & $\mathbf{N}^{\circ}$ & Porcentaje \\
\hline Adecuado & 42 & 60 \\
\hline Sobrepeso & 16 & 23 \\
\hline Obesidad & 12 & 17 \\
\hline Total & 70 & 100 \\
\hline
\end{tabular}

Fuente: Autores.

La caracterización del estrato socioeconómico resultó en que los escolares son procedentes, en un alto porcentaje, del 
@.LIMENTECH CIENCIA Y TECNOLOGÍA ALIMENTARIA ISSN 1692-7125. Volumen 14 No. 2, p. 78 -88, año 2016 Facultad de Ingenierías y Arquitectura Universidad de Pamplona

estrato 1 y 2 , con un total de 49 niños $(70 \%)$ (Ver figura 2).

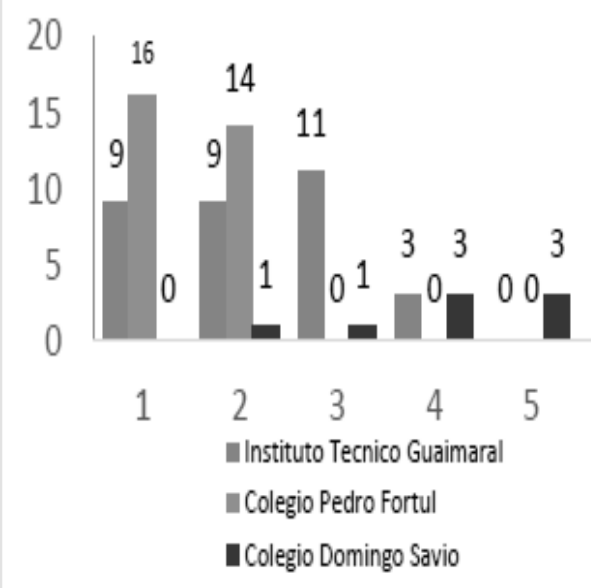

Figura 2. Estrato socioeconómico. Fuente: Autores.

En cuanto a la relación de estrato socioeconómico con sobrepeso y obesidad, se observa que en los estratos 2 y 3 se encuentran en mayor proporción que los demás estratos. (Ver tabla 2).

Tabla 2. Relación estrato socioeconómico y estado nutricional.

\begin{tabular}{|c|c|c|c|c|}
\hline \multirow{2}{*}{ ESTRATO } & \multicolumn{2}{|c|}{ SOBREPESO } & \multicolumn{2}{c|}{ OBESIDAD } \\
\cline { 2 - 5 } & $\mathbf{N}^{\circ}$ & $\%$ & $\mathbf{N}^{\circ}$ & $\%$ \\
\hline 1 & 3 & 19 & 2 & 17 \\
\hline 2 & 6 & 37 & 4 & 33 \\
\hline 3 & 4 & 25 & 4 & 33 \\
\hline 4 & 2 & 13 & 2 & 17 \\
\hline 5 & 1 & 6 & 0 & 0 \\
\hline TOTAL & $\mathbf{1 6}$ & $\mathbf{1 0 0}$ & $\mathbf{1 2}$ & $\mathbf{1 0 0}$ \\
\hline
\end{tabular}

Fuente: Autores.

Se establecen los datos a partir de 70 encuestas demostrándose que el $30 \%$ de los estudiantes refiere no realizar actividad física semanal necesaria para su edad y el $31,3 \%$ de los escolares refiere realizar un promedio de 4-6 horas semanales (ver figura 3).

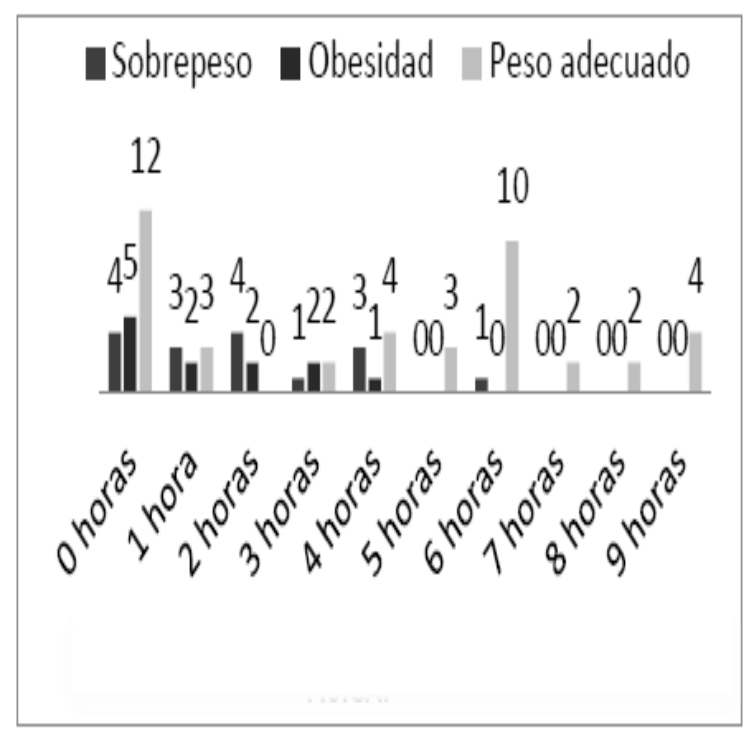

Figura 3. Horas semanales de actividad física. Fuente: Autores.

Con respecto a la población con sobrepeso y obesidad se resalta, que 9 estudiantes no realizan ninguna actividad física diaria y solo un estudiante con sobrepeso realiza 6 .

El $60 \%$ de la totalidad de los estudiantes dedican 1-2 horas a ver televisión, siendo 13 $\%$ de los escolares con sobrepeso, $10 \%$ obesos y $37 \%$ peso adecuado. Con respecto al uso del computador, videojuegos, Smartphone y Tablet se resaltan una similitud puesto que los escolares son sobrepeso y obesidad dedican a estos 
electrodomésticos 1-2 horas diarias observándose mayor inactividad.

En cuanto a las horas de sueño, se encontró que el $77 \%$ de la población duerme un promedio de 8 - 9 horas diarias, 2 escolares con obesidad duermen 6 horas frente a 1 estudiante con peso adecuado ( Ver figura 4).

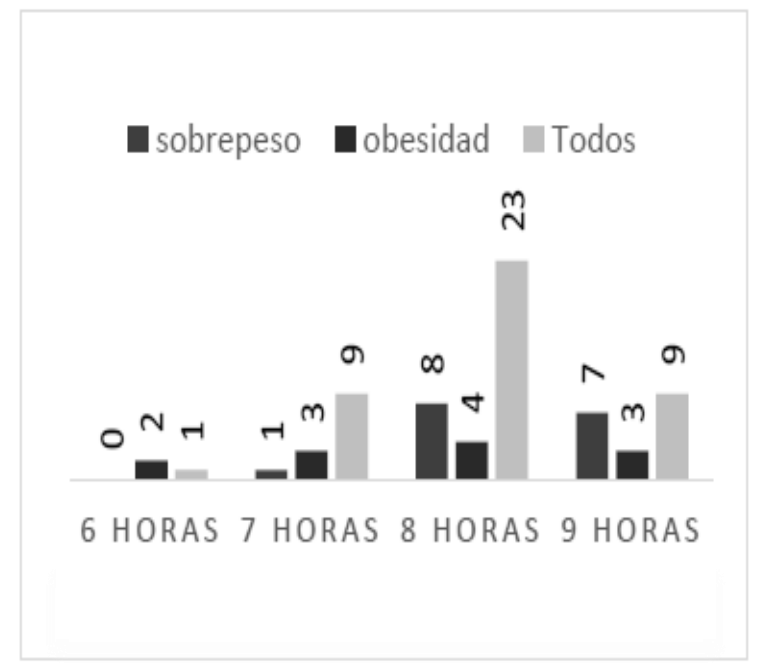

Figura 4. Horas de sueño. Fuente: Autores.

El $31.3 \%$ de los escolares realiza más de 4 a 6 horas de actividad física semanal, en comparación con lo recomendado por la OMS que es mayor de 60 minutos al día (21). El $60 \%$ de los estudiantes empleaban más de 2 horas de tiempo en ocio esto en comparación al estudio de Kristen et al., 2012 donde el empleo mayor de 2 horas en

actividades de ocio se relacionaba con el desarrollo de enfermedades metabólicas (22).

Los estudiantes duermen un promedio de 89 horas en donde la mayoría duermen 8 horas y en base al estudio de Chamorro et. al., Los escolares que duermen menos de 10 horas al día son propensos a sufrir de obesidad en edades futuras (23).

Los estratos socioeconómicos 2 y 3 son los que aportaron un mayor número de estudiantes con sobrepeso y obesidad, esto en comparación con el estudio de Hernández et al., del 2010 donde se demostró en la ciudad de Cartagena el estrato 2 correspondió al estrato con mayor obesidad (24)

De los 70 niños encuestados se determinó que existe una prevalencia de sobrepeso con un porcentaje de $23 \%$ y de obesidad de 17 $\%$, esto con relación a los datos aportados por el ENSIN 2010 en donde se manifiesta que la población infantil la prevalencia de sobrepeso u obesidad es de 25,9\% (25). 


\section{BIBLIOGRAFÍA}

1. Reilly JJ, Wilson D. Obesidad en la niñez. Childhood obesity. BMJ 2006;333;12071210.

2. Álvarez P. Función endocrina en la obesidad. Elsevier Endocrinología y Nutrición 2011, 58 (08).

3. Bueno M. Obesidad infantil. En: Tratado de Endocrinología Pediátrica. Pombo M ed. 3를 Ed. McGraw Hill-Interamericana de España: Madrid, 2002; pp 1247-58.

4. Ministerio de la protección social resolución 2121 de 2010.

5. Serra LI, Aranceta J. Obesidad infantil y juvenil. Estudio enkid (1998-2000). Vol.2 Masson SA: Barcelona 2001.

6. Pallares J, Ayensa J. Estudio de la obesidad infantil y su relación con la imagen corporal en una muestra española. Psychologia. Avances de la disciplina, Vol. 6, N. 2, 2012, pp. 13-21.

7. Chen X, Beydoun MA, Wang Y. Is sleep duration associated with childhood obesity? A systematic review and metaanalysis. Obesity (Silver Spring) 2008; 16:265-74.

8. Zeberio NB. Estudio epidemiológico de obesidad y sobrepeso en niños de ambos sexos de 6 a 10 años del partido de Olavarría, Buenos aires, Argentina. Tesis de maestría en salud pública. Universidad Nacional de la Plata 2010, pp. 1-78.

9. Hirschler V, González C, Cemente G, Talgham S, Petticnichio $H$, Jadzinsky $M$. ¿Cómo perciben las madres de niños de jardín de infantes a sus hijos con sobrepeso? Archivo Argentina de Pediatría 2006;104: (221-226).

10. More RA, Franch A, Gil-Campos M. (2006). Obesidad Infantil. Recomendaciones del Comité de Nutrición de la Asociación Española de Pediatría. Parte I. Prevención. Detección precoz. Papel del pediatra. Anales de Pediatrí, 65:(607-615).

11. Swinburn BA, Caterson I, Seidell J, James WPT. Diet, nutrition and the prevention of excess weight gain and obesity. Public Health Nutrición. 2004, $7:(123-146)$.

12. Pisabarrol $R$, Recalde $A$, Irrasábal $E$, Chaftare Y. ENSO niños 1: Primera encuesta nacional de sobrepeso y obesidad en niños uruguayos. Revista Médica Uruguaya. Diciembre del 2002; 18: (1-6). 
13. Kovalskys I, Bay L, Rausch Herscovici C, Berner E. (2005). Prevalencia de obesidad en una población de 10 a 19 años en la consulta pediátrica. Revista Chilena Pediátrica;76: (324-325).

14. Travé T, Sánchez F. (2005). Obesidad Infantil: ¿Un problema de educación individual, familiar o social?. Acta Pediátrica Española; 63:(204-207).

15. Field A. E, Cook NR, and Gillman MW. (2005). Weight Status in Childhood as a Predictor of Becoming Overweight or Hypertensive in Early Adulthood. The North American Association for the Study of Obesity;13: (163-169).

17. OMS. (2015). Estrategia mundial sobre régimen alimentario, actividad física y salud. ¿Por qué son importantes el sobrepeso y la obesidad infantiles?. Organización Mundial de la Salud (OMS).

18. Rivera J. (Childhood and adolescent overweight and obesity in Latin America: a systematic review. Lancet Diabetes Endocrinol 2013).

19. OMS: Obesidad y sobrepeso, datos y cifras. Organización Mundial de la Salud (OMS), junio 2016, N³11.

20. González M. (2015). Sobrepeso y Obesidad en Adolescentes de Saltillo, Coahuila, México: asociación con variables clínicas, bioquímicas e hígado graso y una propuesta de intervención educativa integral. Tesis doctoral en internet. Barcelona, Universidad de Barcelona.

21. OMS. (2016). La actividad física en los jóvenes.

22. Herrick KA, Fakhouri THI, Carlson SA, Fultron JE. (2012). TV Watching and Computer Use in U.S. Youth Aged 12-15, NCHS Data brief. 2014;154:1-8.

23. Chamorro R, Durán S, Reyes S, Ponce R, Algarín C, Peirano P. La reducción del sueño como factor de riesgo para obesidad. Rev méd Chile. 2011;139(7):932-940.

24. Guiomar María Hernández Álvarez. prevalencia de sobrepeso y obesidad, y factores de riesgo, en niños de 7-12 años, en una escuela pública de Cartagena Septiembre - Octubre DE 2010, Op. Cit., pp86.

25. Encuesta Nacional de la Situación Nutricional en Colombia 2010. 